\title{
A large Wolf-Rayet population in NGC 300 uncovered by VLT-FORS2 ${ }^{\star}$
}

\author{
H. Schild ${ }^{1}$, P. A. Crowther ${ }^{2}$, J. B. Abbott ${ }^{2}$, and W. Schmutz ${ }^{3}$ \\ 1 Institut für Astronomie, ETH-Zentrum, 8092 Zürich, Switzerland \\ 2 Dept of Physics and Astronomy, University College London, Gower St, London WC1E 6BT, UK \\ ${ }^{3}$ Physikalisch-Meteorologisches Observatorium, 7260 Davos, Switzerland
}

Received 6 September 2002 / Accepted 25 October 2002

\begin{abstract}
We have detected 58 Wolf-Rayet candidates in the central region of the nearby spiral galaxy NGC 300, based on deep VLT-FORS2 narrow-band imaging. Our survey is close to complete except for heavily reddened WR stars. Of the objects in our list, 16 stars were already spectroscopically confirmed as WR stars by Schild \& Testor and Breysacher et al., to which 4 stars are added using low resolution FORS2 datasets. The WR population of NGC 300 now totals 60 , a threefold increase over previous surveys, with $\mathrm{WC} / \mathrm{WN} \geq 1 / 3$, in reasonable agreement with Local Group galaxies for a moderately sub-solar metallicity. We also discuss the WR surface density in the central region of NGC 300. Finally, analyses are presented for two apparently single WC stars - \#29 (alias WR3, WC5) and \#48 (alias WR13, WC4) located close to the nucleus, and at a deprojected radius of $2.5 \mathrm{kpc}$, respectively. These are among the first models of WR stars in galaxies beyond the Local Group, and are compared with early WC stars in our Galaxy and LMC.
\end{abstract}

Key words. galaxies: individual: NGC 300 - stars: Wolf-Rayet - stars: fundamental parameters

\section{Introduction}

Over 500 Wolf-Rayet stars have been identified in Local Group galaxies, principally the Milky Way, M 31 and M 33. These stars beautifully trace young stellar populations, and their number and distribution reacts sensitively to metallicity, which varies by an order of magnitude from the Small Magellanic Cloud (SMC) to M 31. Detailed studies of individual WR stars in Local Group stars have been carried out (e.g. Smartt et al. 2001; Crowther 2000; Crowther et al. 2002) using 2-4 m class telescopes.

The availability of $8-10 \mathrm{~m}$ class telescopes permits the discovery and study of individual stars at greater distances, spanning a greater range of metallicities. As a first application, we present here VLT imaging and spectroscopy of WR stars in NGC 300, located in the Sculptor group at a distance of $2 \mathrm{Mpc}$ (Freedman et al. 2001). It's metallicity is bracketed by the Milky Way and Large Magellanic Cloud (LMC) and therefore we expect a similarly large number of WR stars in NGC 300. Previous surveys have however failed to identify them. A large population might also be anticipated since NGC 300 is a late type spiral, reminiscent of M 33, which harbours at least 140 WR stars (Massey \& Johnson 1998). Because of its low inclination NGC 300 is well suited to studies

Send offprint requests to: $\mathrm{P}$. A. Crowther,

e-mail: pac@star.ucl.ac.uk

* Based on observations made with ESO Telescopes at the Paranal Observatory under programme ID 65.H-0705(A). of its stellar content - recent surveys include blue supergiants (Bresolin et al. 2002a), Cepheids (Pietrzyński et al. 2002b), OB associations (Pietrzyński et al. 2001) and Supernova remnants (Pannuti et al. 2001).

The first signature of WR stars in NGC 300 was found in spectra of H II regions by D'Odorico et al. (1983). They detected the broad WR feature in two out of sixteen $\mathrm{H}$ II regions. Five years later, Deharveng et al. (1988) presented a catalogue of $176 \mathrm{H}$ II regions and found broad WR emission in four of them. Although this clearly demonstrated that WR detection was feasible at this distance, the spectroscopy could not in itself locate the individual WR stars. To achieve this, imaging in narrow band filters was necessary. First results with this technique were reported by Schild \& Testor $(1991,1992)$ and Testor \& Schild (1993). They found in total 13 WR candidates and confirmed them spectroscopically. Six additional WR stars were later identified in the same way by Breysacher et al. (1997) in stellar associations. One additional weak-lined late WN star was serendipitously found by Bresolin et al. (2002ab).

In total, there are presently 22 confirmed WR stars in NGC 300, strongly skewed towards WC subtypes. From a census of the WR distribution in Local Group galaxies, Massey (1996) identified a rather tight correlation between the WC/WN ratio and metallicity, as characterized by oxygen content. Although there have not been any recent studies of the NGC 300 metallicity gradient, Deharveng et al. (1988) used data from Pagel et al. (1979) and Webster \& Smith (1983), to imply a range between $\log (\mathrm{O} / \mathrm{H})+12=8.9$ in its nucleus and 

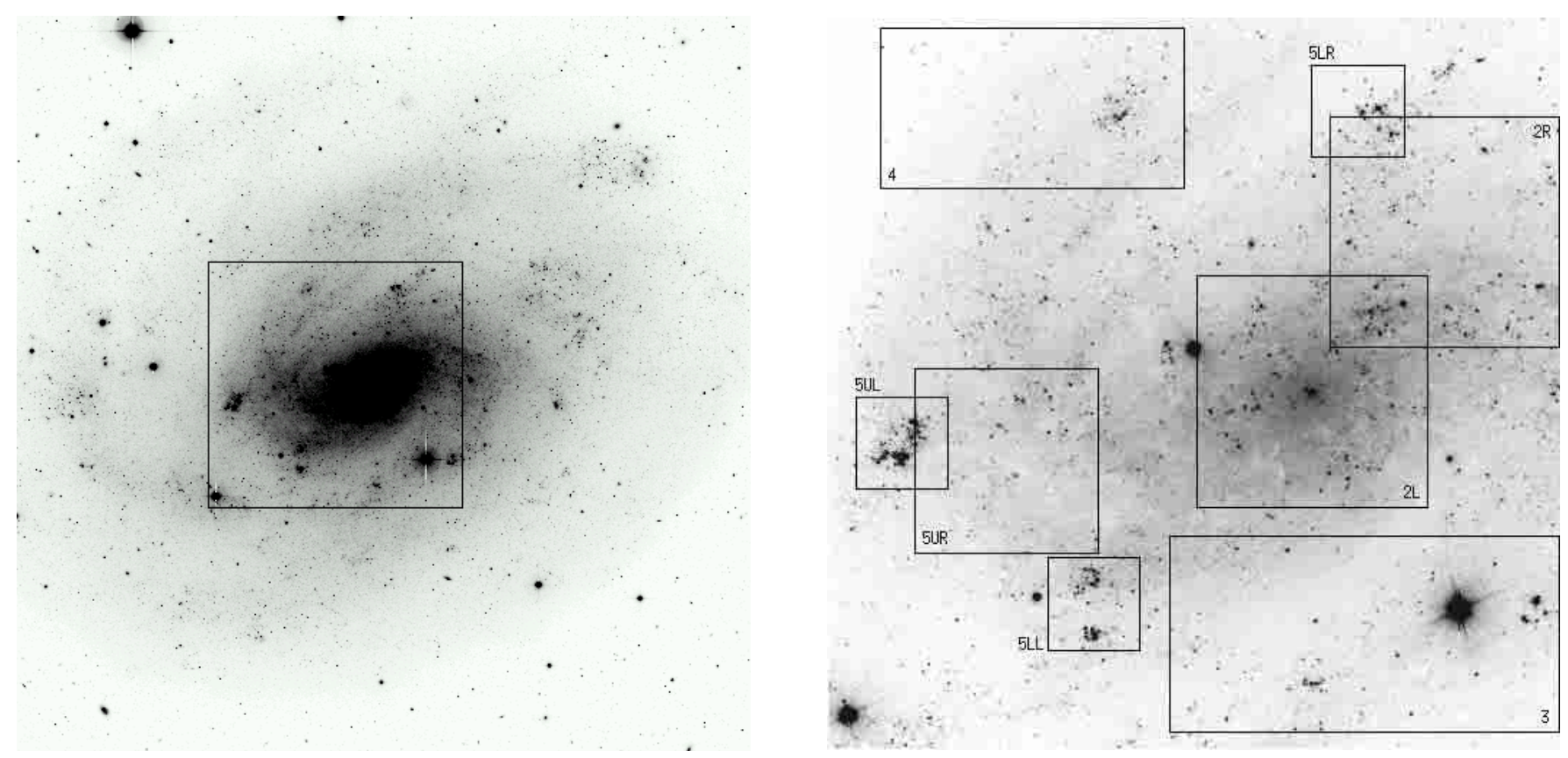

Fig. 1. Left: $R$-band ESO $2.2 \mathrm{~m}$ WFI image (see http: //www. eso.org/outreach/press-rel/pr-2002/phot-18-02 .html), with a 20' $\times$ $20^{\prime}$ field-of-view, corresponding to the isophotal diameter of NGC 300. The position of the adjacent FORS2 frame is marked. Right: $\lambda 4684$ narrow-band filter FORS2 frame $\left(6.8^{\prime} \times 6.8^{\prime}\right)$ showing the location of subsequent finding charts in NGC $300(\mathrm{~L}=$ left, LL = lower left, $\mathrm{UL}=$ upper left, $\mathrm{R}=$ right, $\mathrm{LR}=$ lower right, $\mathrm{UR}=$ upper right). North to the top, East to the left.

8.3 in its outer spiral arms. Similar conclusions were obtained by Zaritsky et al. (1994) from a recalibration of previous results. One would expect a WC/WN ratio of $\sim 1 / 2$ from comparison with Local Group galaxies, yet the census of WR stars in NGC 300 indicates WC/WN 2. Consequently, we might expect that the WR population of NGC 300 is highly incomplete, particularly amongst WN stars.

In this paper we present results from a new imaging survey of the central region of NGC 300 with the Very Large Telescope (VLT). New WR candidates are identified, some of which are spectroscopically confirmed. Spectral types of the latter are discussed, with particular reference to the WC/WN ratio of the inner galaxy. An analysis of two apparently single WC stars is presented, one located close to its nucleus, the other at $\sim 50 \%$ of the Holmberg radius, $\rho_{0}$. Comparisons are made with recent comparable studies of WC stars in a variety of metallicity environments.

\section{Observations}

We observed NGC 300 with the VLT UT2 (Kueyen) and Focal Reduced/Low Dispersion Spectrograph \#2 (FORS2) during 2000 September $2-3$. The conditions were photometric but the seeing was highly variable, changing from 0.6 to $3.5^{\prime \prime}$ and we used the instrument accordingly in imaging and spectroscopic mode.

\subsection{Imaging}

While the seeing was good (typically $0.8^{\prime \prime}$ ) we obtained images through two interference filters with central wavelengths at $4684 \AA$ and $4781 \AA$ and band widths of $66 \AA$ and $68 \AA$, respectively. The former filter is well matched to the strong WR emission feature containing the $\mathrm{N}_{\text {III }} \lambda 4640, \mathrm{C}_{\text {III }} \lambda 4650$, C IV $\lambda 4658$ and He II $\lambda 4686$ emission lines. The wavelength range of the latter filter falls into a spectral region that is free from emission lines. We collected two images in each filter with exposure times of $600 \mathrm{~s}$. These frames were centred at $\alpha$ :

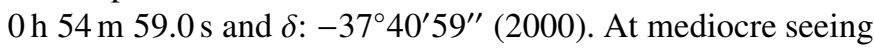
conditions short exposures through Bessel $B$ and $V$ filters were also collected. Only one of the $V$ frames was of sufficient quality, but it was slightly offset such that only $V$-band magnitudes of WR candidates with RA larger than $00 \mathrm{~h} 54 \mathrm{~m} 46.7 \mathrm{~s}$ could be measured.

The standard collimator was used, providing a field-of-view of $6.8^{\prime} \times 6.8^{\prime}$ with an image scale of $0.2^{\prime \prime} /$ pixel. The detector was a $2048 \times 2048$ Tektronix CCD with $24 \mu \mathrm{m}$ pixels. The left panel of Fig. 1 illustrates the region of NGC 300 covered by FORS2, using an archival ESO $2.2 \mathrm{~m}$ Wide Field Imager (WFI) $R$-band frame, obtained in reasonable seeing (1.1 arcsec), and kindly provided by M. Schirmer. An overview of the $\lambda 4684$ narrow-band filter FORS2 image is shown in the right panel of Fig. 1, which indicates the regions covered by subsequent finding charts.

The data were de-biased and flat fielded with frames taken in the following morning twilight. We used the DAOPHOT software package to get relative photometry. These were converted into absolute fluxes with the photometric standard stars in the field of NGC 300 listed in Pietrziński et al. (2002a).

\subsection{Spectroscopy}

FORS2 was used in long slit mode (LSS) to obtain spectroscopy for selected Wolf-Rayet stars and candidates. 

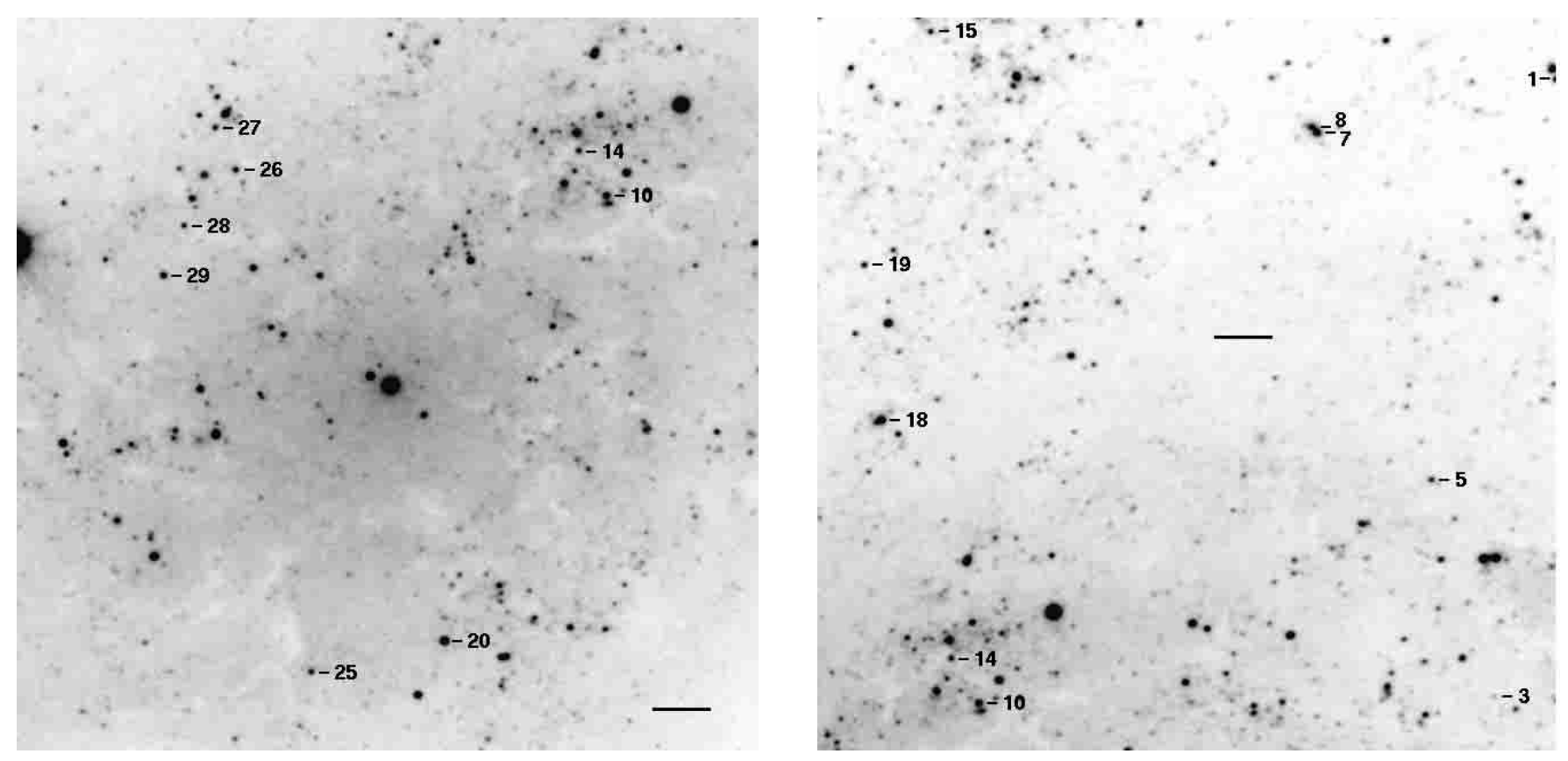

Fig. 2. Finding chart for WR stars/candidates in NGC 300. Left: nuclear region, right: area northwest of the nucleus. The horizontal bar represents $10^{\prime \prime}$. North to the top, East to the left.

This mode was selected since narrow-band images were not obtained in advance of the observing run. The $300 \mathrm{~V}$ grating, GG435 filter and 1.0" slit width provided spectroscopy covering $\lambda \lambda 3500-8970$ at a dispersion of $1.7 \AA /$ pixel, corresponding to a resolution of $R=\Delta \lambda / \lambda \sim 440$ at $5900 \AA$. Eight targets were observed with this configuration using $1800 \mathrm{~s}$ exposures, and will be discussed in Sect. 4. Generally, two WR stars were observed in each observation via a suitable choice of position angle using the FORS Instrument Mask Simulator (FIMS) software. Relative flux calibration was achieved using short exposures for standard stars Feige 110 and LTT 1788. Absolute calibration required convolution with $b$ and $v$ (Smith 1968) narrow-band filters, which were approximated to our $m_{4781}$ photometry.

For three stars, higher resolution 600R grating observations were obtained, using the GG435 filter, covering $\lambda \lambda 5330-7540$, at a dispersion of $0.7 \AA /$ pixel, corresponding to a resolution of $R \sim 1000$ at $6300 \AA$. Two $1800 \mathrm{~s}$ exposures were taken before seeing conditions deteriorated. Identical flux standards were again used with this configuration. For all datasets a standard data reduction was carried out using FIGARO $^{1}$ i.e., bias subtraction, flat field correction, extraction, wavelength and flux calibration.

\section{Wolf-Rayet candidates}

\subsection{New catalogue}

Our frames were positioned about one arcminute East of the centre of NGC 300 such that they covered the bright HII

\footnotetext{
1 www.starlink.rl.ac.uk/star/docs/sun86.htx/ sun86.html
}

regions Deh 53 and 137 (Deharveng et al. 1988). Therefore, we have surveyed the central region of the galaxy, but do not include the outer spiral arms beyond a Holmberg radius $\rho_{0} \gtrsim 0.4-0.5$, where $\rho_{0}=9.75^{\prime} \sim 5.75 \mathrm{kpc}$ (recall Fig. 1 ). The sensitivity by which WR emission features can be detected obviously depends on the signal/noise ratio. In this case the signal is the flux difference between on-off frames. We first identified the $\lambda 4684$ emission objects on the difference image. The selection criteria were a stellar appearance and a peak intensity of at least $6 \sigma$. The probability for any of the listed objects of having indeed a WR excess is therefore very high. For these objects we subsequently picked the $V$, emission ( $\lambda 4684)$ and continuum ( 14781$)$ magnitudes from the (rather long) DAOPHOT photometric list.

It should be noted that photometry is hampered by a variable background due to unresolved galaxy emission and heavy crowding, which can be particularly severe in OB associations. It follows that while the identification as a $\lambda 4684$ emission object is rather reliable, the quantitative measurement of a WR excess is less certain. Our narrow-band images are complete down to 23.7 mag while the $3 \sigma$ detection limit was at $24.7 \mathrm{mag}$.

We present a catalogue of the $58 \mathrm{WR}$ stars/candidates identified in our images in Table 1. We include spectral types taken from the literature, updated in case of revisions from our new spectroscopy (see Sect. 4), plus associated H II regions from Deharveng et al. (1988). De-projected galactocentric distances were calculated using parameters from Table 1 of Deharveng et al. (1988). In Figs. 2 to 5 we give finding charts for the WR candidates. In all of them a horizontal bar is plotted that represents $10^{\prime \prime}$. The orientation is as usual: North to the top and East to the left. All finding charts are from our $\lambda 4684$ narrow-band filter images. 
Table 1. List of Wolf-Rayet stars and candidates in the central regions of NGC 300. Two other WR stars are known outside the present field WR8 (WN) in Deh 30 from Schild \& Testor (1992) and a WC in Deh 24 from D'Odorico et al. (1983). Deprojected galactocentric distances are expressed as a fraction of the Holmberg radius $\left(\rho_{0}=9.75^{\prime} \simeq 5.75 \mathrm{kpc}\right)$.

\begin{tabular}{|c|c|c|c|c|c|c|c|c|c|c|c|}
\hline & RA (J2000) & Dec (J2000) & $m_{4684}$ & $m_{4781}-m_{4684}$ & $m_{V}$ & $\rho / \rho_{0}$ & $\operatorname{Deh}^{4)}$ & Spect Type & $\mathrm{ST}^{1)}$ & Brey $^{2)}$ & Remark \\
\hline 1 & 05441.7 & -373839.0 & out & out & out & 0.44 & 48 & WC4 & & & 5) \\
\hline 2 & 05442.37 & -37437.3 & 18.93 & 1.02 & out & 0.33 & $53 b$ & WC4 & WR 10 & & \\
\hline 3 & 05442.61 & -374028.5 & 21.88 & 1.25 & out & 0.25 & & & & & \\
\hline 4 & 05442.78 & -37431.8 & 17.88 & 0.25 & out & 0.32 & $53 \mathrm{c}$ & WN & & & 4) \\
\hline 5 & 05443.53 & -373949.9 & 20.52 & 2.09 & out & 0.28 & 54 & & & & \\
\hline 6 & 05444.75 & -374240.0 & 19.03 & 0.13 & out & 0.26 & & WN11 & & & 3) \\
\hline 7 & 05445.22 & -373848.0 & 19.71 & 0.14 & out & 0.37 & 61 & & & & \\
\hline 8 & 05445.28 & -373847.0 & 20.22 & 0.24 & out & 0.37 & 61 & & & & \\
\hline 9 & 05447.69 & -374245.3 & 20.26 & 0.58 & 20.62 & 0.24 & & WN4-5 & & & 5) \\
\hline 10 & 05450.21 & -374029.7 & 19.68 & 0.52 & 20.04 & 0.10 & $76 a$ & & & & \\
\hline 11 & 05450.22 & -373824.1 & 18.51 & 0.44 & 18.79 & 0.37 & 77 & $\mathrm{WC}+\mathrm{WN}$ & WR 9 & $\mathrm{I}-1$ & \\
\hline 12 & 05450.53 & -373826.7 & 20.87 & 1.57 & 22.12 & 0.36 & 77 & & & $\mathrm{I}-2$ & \\
\hline 13 & 05450.57 & -373813.2 & 20.54 & 0.29 & 20.58 & 0.39 & & & & & \\
\hline 14 & 05450.62 & -374021.7 & 20.39 & 1.49 & 21.64 & 0.11 & $76 \mathrm{~b}$ & WC4-6 & WR 1 & & \\
\hline 15 & 05450.91 & -373830.0 & 20.35 & 0.16 & 20.41 & 0.35 & 79 & & & & \\
\hline 16 & 05451.00 & -373826.1 & 20.10 & 0.10 & 20.27 & 0.36 & 79 & & & & \\
\hline 17 & 05451.31 & -373826.3 & 22.50 & 0.14 & weak & 0.36 & 79 & & & & \\
\hline 18 & 05451.65 & -373939.2 & 19.11 & 0.10 & 18.79 & 0.19 & 84 & & & & \\
\hline 19 & 05451.89 & -373911.5 & 20.20 & 0.08 & 19.24 & 0.25 & & & & & \\
\hline 20 & 05452.59 & -374149.1 & 18.54 & 0.11 & 17.57 & 0.11 & 85 & & & & \\
\hline 21 & 05452.93 & -373838.3 & 20.85 & 0.12 & 20.97 & 0.32 & & & & & \\
\hline 22 & 05453.09 & -374334.0 & 20.98 & 1.16 & 21.71 & 0.35 & 87 & WC4 & & & 5) \\
\hline 23 & 05453.11 & -374347.3 & 19.72 & 0.08 & 19.71 & 0.38 & 88 & & & & \\
\hline 24 & 05453.80 & -374347.2 & 20.00 & 1.55 & 21.34 & 0.38 & 90 & WC5 & WR 11 & & \\
\hline 25 & 05454.57 & -374154.7 & 20.28 & 0.09 & 20.27 & 0.13 & & & & & \\
\hline 26 & 05455.67 & -374025.1 & 20.12 & 0.08 & 20.10 & 0.09 & & & & & \\
\hline 27 & 05455.99 & -374017.6 & 20.39 & 0.15 & 20.47 & 0.11 & 96 & & & & \\
\hline 28 & 05456.45 & -374035.1 & 20.55 & 1.90 & 22.05 & 0.09 & 98 & WNE & WR 2 & & \\
\hline 29 & 05456.76 & -374044.0 & 19.71 & 2.58 & 21.61 & 0.08 & 98 & WC4-5 & WR 3 & & \\
\hline 30 & 05458.95 & -374358.7 & 21.24 & 1.31 & 22.01 & 0.44 & 107 & WN & & & 5) \\
\hline 31 & $055 \quad 0.65$ & -373851.5 & 20.74 & 1.68 & 22.14 & 0.31 & & & & & \\
\hline 32 & $055 \quad 2.33$ & -373827.4 & 18.84 & 0.08 & 18.84 & 0.37 & 115 & & & & \\
\hline 33 & $\begin{array}{l}0552.88\end{array}$ & -374316.0 & 21.28 & 1.02 & 21.51 & 0.40 & & & & II-1 & \\
\hline 34 & 0553.34 & -374242.0 & 20.36 & 1.97 & 21.87 & 0.35 & & WC5-6 & WR 12 & III-1 & \\
\hline 35 & 0553.55 & -374249.4 & 19.06 & 0.09 & 18.82 & 0.36 & & & & & \\
\hline 36 & 0553.64 & -374320.0 & 18.06 & 0.13 & 17.97 & 0.42 & & & & & \\
\hline 37 & 0553.75 & -374251.6 & blend & 2.0 & 18.56 & 0.37 & & & & & \\
\hline 38 & 0554.09 & -374318.9 & 19.07 & 0.64 & 19.53 & 0.43 & & WN9-10 & WR 7 & III-2 & \\
\hline 39 & 0554.17 & -374316.6 & 20.05 & 0.08 & 19.99 & 0.42 & & & & & \\
\hline 40 & 0555.69 & -374113.4 & 20.13 & 1.68 & 21.56 & 0.28 & & WCE & WR 6 & & \\
\hline 41 & 0559.98 & -374212.5 & 21.64 & 0.95 & 22.44 & 0.43 & & & & & \\
\hline 42 & 05511.03 & -373755.5 & 22.02 & 0.56 & 22.39 & 0.52 & & & & & \\
\hline 43 & 05512.07 & -374121.9 & 19.84 & 0.12 & 19.83 & 0.42 & $137 \mathrm{~d}$ & & & & \\
\hline 44 & 05512.19 & -374119.7 & 17.71 & 0.19 & 17.80 & 0.42 & $137 d$ & & & & \\
\hline 45 & 05512.21 & -374120.4 & 18.55 & 0.40 & 18.79 & 0.42 & $137 d$ & & & IV-1 & \\
\hline 46 & 05512.32 & -374138.4 & 20.53 & 1.89 & 21.69 & 0.44 & $137 \mathrm{a}$ & WC4-5 & & $\mathrm{V}-1$ & \\
\hline 47 & 05512.41 & -374129.0 & 19.68 & 2.95 & 21.19 & 0.43 & $137 b$ & WC5-6 & WR 5 & IV-2 & \\
\hline 48 & 05512.54 & -374123.6 & 20.23 & 3.31 & 21.87 & 0.43 & $137 \mathrm{~b}$ & WC4 & WR 13 & IV-3 & \\
\hline 49 & 05512.58 & -374139.5 & 18.46 & 0.31 & 18.85 & 0.45 & $137 \mathrm{a}$ & WN7 & WR 4 & $\mathrm{~V}-2$ & \\
\hline 50 & 05512.65 & -374138.5 & 19.62 & 0.18 & 19.73 & 0.45 & $137 \mathrm{a}$ & & & & \\
\hline 51 & 05512.72 & -374144.6 & blend & 0.7 & 20.38 & 0.45 & $137 \mathrm{a}$ & & & & \\
\hline 52 & 05513.18 & $\begin{array}{lll}-3738 & 1.4\end{array}$ & 22.16 & 0.5 & 22.74 & 0.54 & & & & & \\
\hline 53 & 05513.23 & -374139.8 & 20.63 & 1.12 & 21.54 & 0.46 & & WN4-6 & & $\mathrm{V}-3$ & \\
\hline 54 & 05513.36 & -374130.3 & 20.22 & 0.30 & 20.39 & 0.46 & & & & & \\
\hline 55 & 05513.47 & -374146.3 & 20.64 & 1.87 & 22.30 & 0.47 & & WN4-5 & & V-4 & \\
\hline 56 & 05513.51 & -374137.9 & 17.73 & 0.11 & 17.68 & 0.46 & $137 \mathrm{c}$ & & & & \\
\hline 57 & 05513.61 & -374132.0 & 20.31 & 0.11 & 20.34 & 0.46 & & & & & \\
\hline 58 & 05513.93 & -374143.2 & 18.82 & 0.08 & 18.75 & 0.48 & $137 \mathrm{c}$ & & & & \\
\hline
\end{tabular}

1) Schild \& Testor (1991, 1992), Testor \& Schild (1993); ${ }^{2)}$ Breysacher et al. (1997); ${ }^{3)}$ Bresolin et al. (2002ab); ${ }^{4)}$ Deharveng et al. (1988);

5) This paper. 


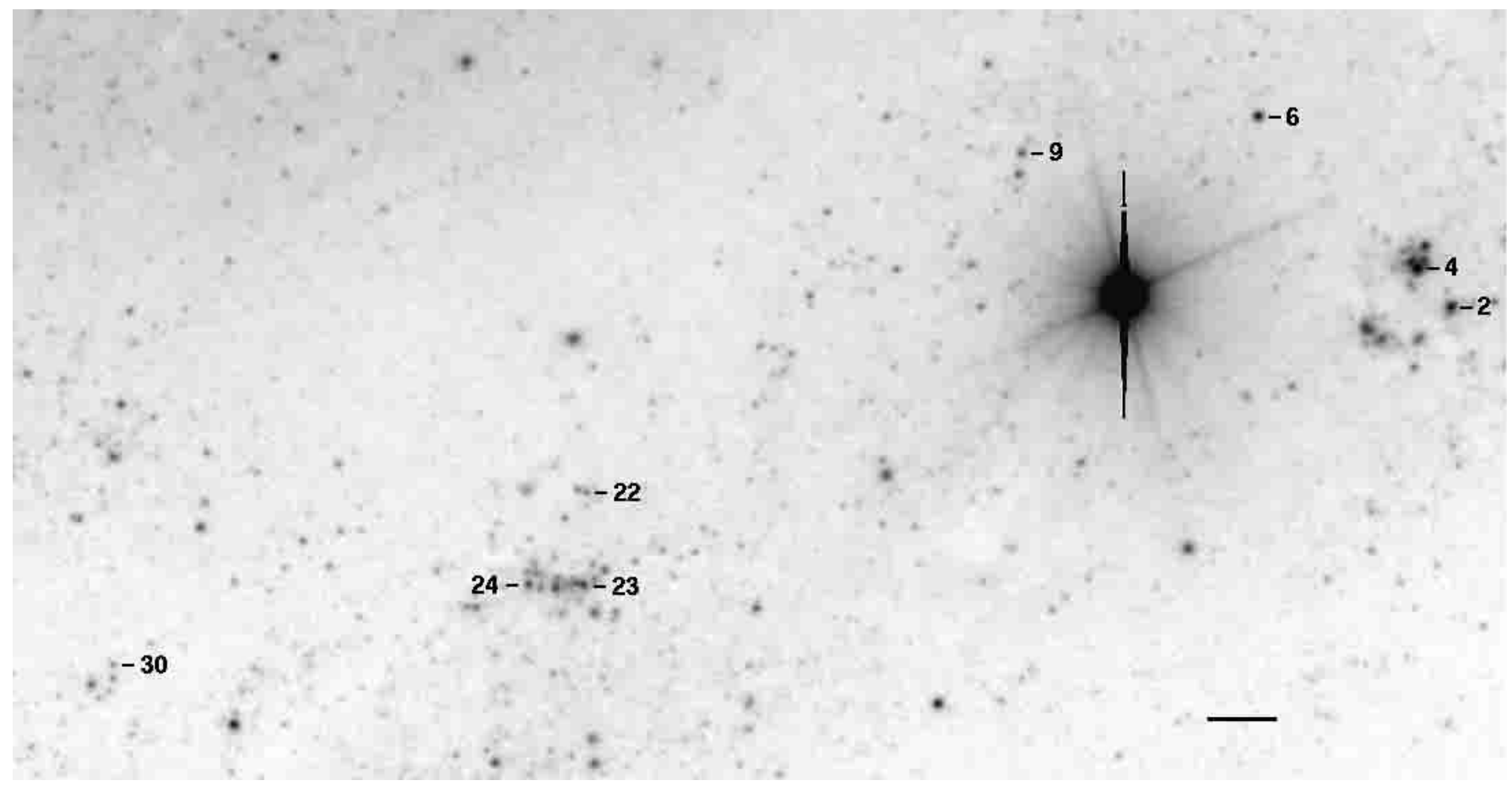

Fig. 3. Finding chart for WR stars/candidates in the southern spiral arm. The saturated object is the galactic foreground star CD-38 301 . The horizontal bar represents $10^{\prime \prime}$. North to the top, East to the left.

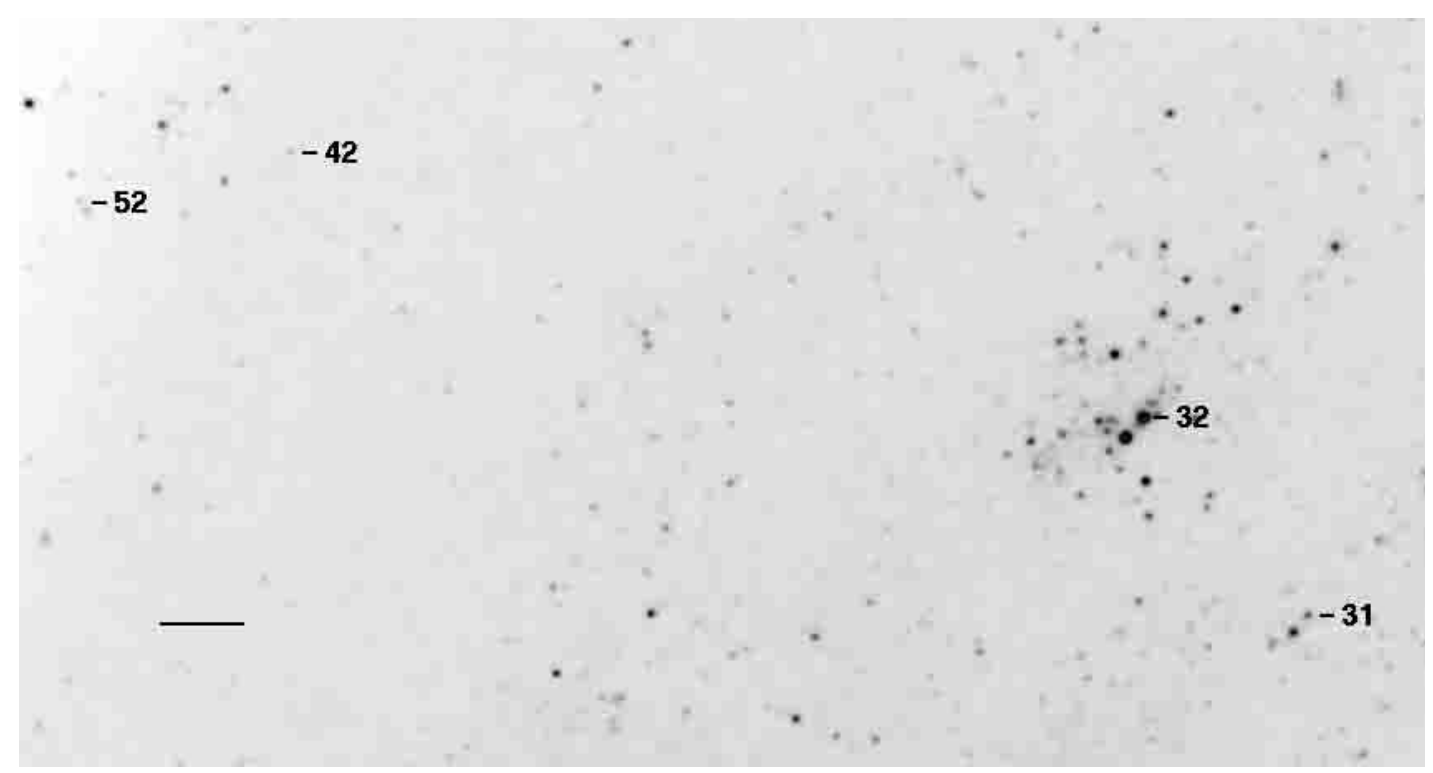

Fig. 4. Finding chart for WR stars/candidates in the northeast of the nucleus. Some vignetting occurred in the upper left corner (northeast). The horizontal bar represents $10^{\prime \prime}$. North to the top, East to the left.

\subsection{Nature of candidates}

39 out of our 58 WR candidates with a $\lambda 4684$ excess are newly identified in this study. We compare the $\lambda 4781$ continuum magnitudes with the $\lambda 4684$ excess in Fig. 6. Stars with previous (or new) spectroscopic confirmation are indicated, as shown in the key. We include estimates of the absolute magnitudes at $\lambda 4781$, assuming a distance modulus of 26.53 mag to NGC 300 (Freedman et al. 2001) and $A_{\lambda 4781}=0.36 \mathrm{mag}$, corresponding to $E_{B-V}=0.10 \mathrm{mag}$ which is the mean interstellar reddening towards H II regions of NGC 300 within $\rho / \rho_{0}=0.5$ derived by Deharveng et al. (1988).

Figure 6 clearly separates the large $\lambda 4684$ excesses of the visually faint WC and early-type WN stars, from the small excesses of the visually bright late-type WN stars and WR binaries. Approximate line equivalent widths (in $\AA$ ) are also presented in the figure, as estimated from stars in which optical spectroscopy is available (see also Fig. 4 of Massey \& Johnson 1998). Such comparisons would represent the sole means by which WR populations might be identified in galaxies which 

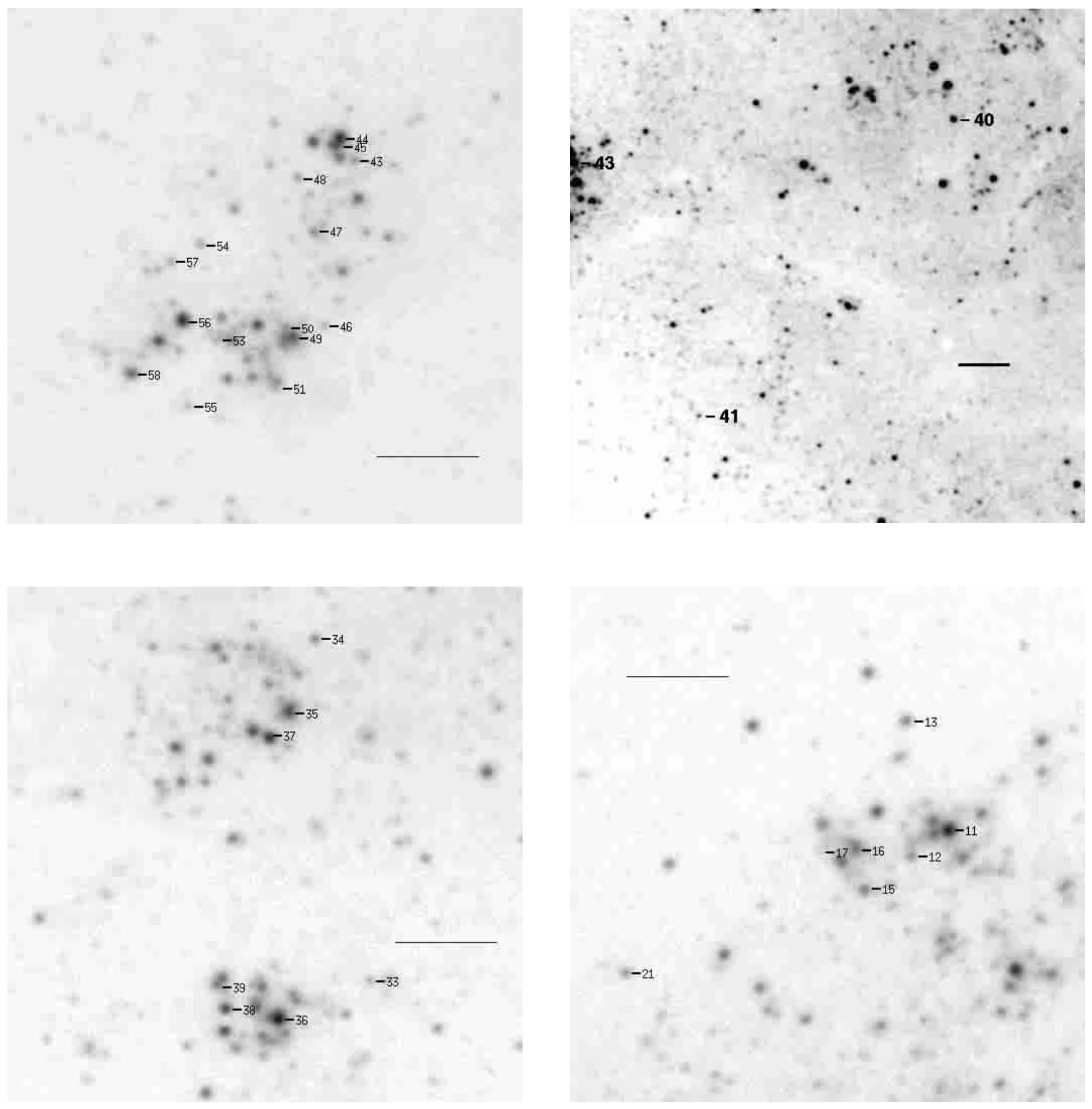

Fig. 5. Finding chart for WR stars/candidates east of the nucleus (top right) and int he H ir regions Deh 137 (top left), Deh 118/119 (bottom left) and Deh 77/79 (bottom right). The horizontal bars represent 10". North to the top, East to the left.

are too distant (or reddened) for confirmatory spectroscopy to be obtained, even with large 8-10 m telescopes.

Previously known WR stars tend to have large $\lambda 4684$ excesses of $\gtrsim 1 \mathrm{mag}$, corresponding to emission equivalent widths of $\gtrsim 100 \AA$. Exceptions include those WR stars which lie in well surveyed OB associations. We find six more WR candidates with such a large WR excess (\#3, \#5, \#22, \#30, \#31 and \#37). They are rather faint with $m_{4684}>20.5 \mathrm{mag}$, which is presumably why they escaped earlier detection. From this sample, \#22 and \#30 were observed spectroscopically, such that both were confirmed as WR stars - see Sect 4.
Single, early-type WC stars have $24650-4686$ emission equivalent widths of $\gtrsim 1000 \AA$, and are visually rather faint. Consequently only three WC stars, with an excess of $\geq 2.5 \mathrm{mag}$ are likely to be single, namely \#29, \#47 and \#48. Two of these stars are discussed in detail in Sect. 5. Other WC stars are almost certainly multiple, or suffer contamination from stars along the same line-of-sight. Early-type WN stars possess He II $\lambda 4684$ emission equivalent widths of $100-400 \AA$, corresponding to excesses of 1-2 mag. From Fig. 6, it is likely that \#28 and \#55 are single, whilst others are probably multiple. For those remaining WR candidates with excesses greater than 1 mag, \#5 


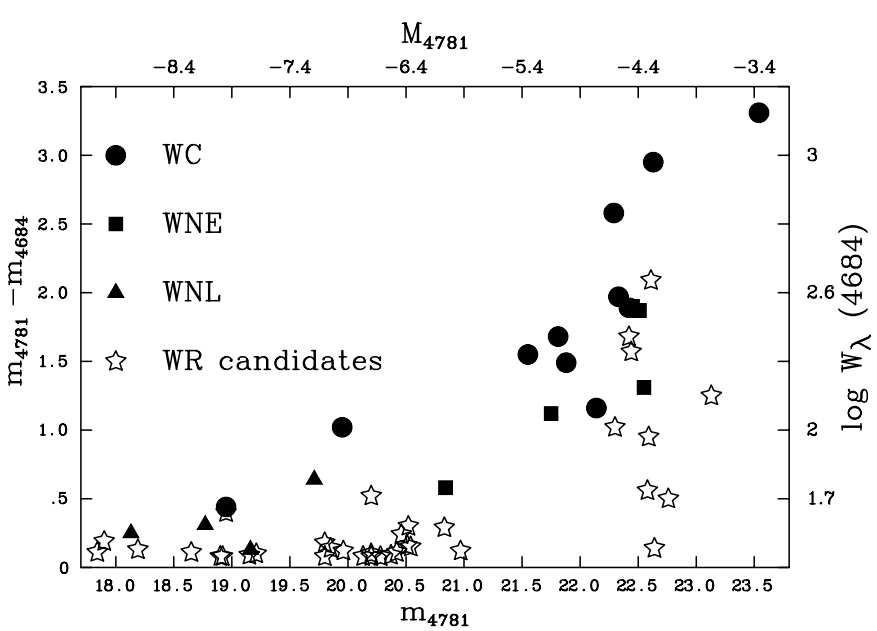

Fig. 6. Comparison between the $\lambda 4781$ continuum magnitudes of the WR candidates in NGC 300 and the $\lambda 4684$ excess (stars). Spectroscopically confirmed WR stars are presented in the key. Approximate absolute magnitudes and line equivalent widths are indicated, as discussed in the text. Three WR stars are not shown in this figure - \#1 (located at the edge of the image surveyed - Fig. 2), \#37 and \#51 (both are severely blended).

is a strong $\mathrm{WC}+\mathrm{O}$ candidate, whilst the remainder are probable $\mathrm{WN}+\mathrm{OB}$ systems.

There are also a handful of new WR candidates with an excess in the range 0.3 to $1 \mathrm{mag}(\# 9, \# 10, \# 41, \# 42, \# 51, \# 52$ and \#54), corresponding to emission equivalent widths in the range $\sim 30-100 \AA$. Their relatively small line strengths suggests that if they are visually bright, with $M_{4781}<-6$, they are WR binaries (e.g. \#11) or single WN7-9 stars (e.g. \#38). If they are visually faint, they are probably weak-lined single WN stars (e.g. \#41). From this group, \#9 was observed spectroscopically and confirmed as a WN star - see Sect. 4. Numbers 51 and 54 lie in the giant $\mathrm{H}_{\text {II }}$ region Deh 137 while \#10 is another WR object in the nuclear area of NGC 300 (Fig. 2).

25 WR candidates have a $\lambda 4684$ excess below 0.3 mag. It is possible that some of these stars are borderline WR/Of stars. Except in one case, they are visually bright, with $M_{4781}<-6$, as expected for a WR binary, single late WN star, or an extreme O-type supergiant, with strong He II $\lambda 4686$ emission (i.e. an Of star). Indeed, \#6 was independently found by Bresolin et al. (2002a) and classified as a WN 11 star. Our $\lambda 4684$ excess of 0.13 mag is in close agreement with the spectroscopy of Bresolin et al. (2002b). This indicates that a He II excess of $\sim 0.1$ mag can be reliably measured with this instrumentation, and hence also that Of stars and very late WN stars can easily be detected. Most candidates from the present sample are probably WR rather than Of since, since by definition, He II $\lambda 4686$ equivalent widths of the latter do not exceed $\sim 12 \AA$ (Bohannan \& Crowther 1999).

\subsection{Completeness, surface density and the WC/WN ratio}

In addition to verifying the likely-hood of whether our candidates are genuine WR stars, how complete is our survey?

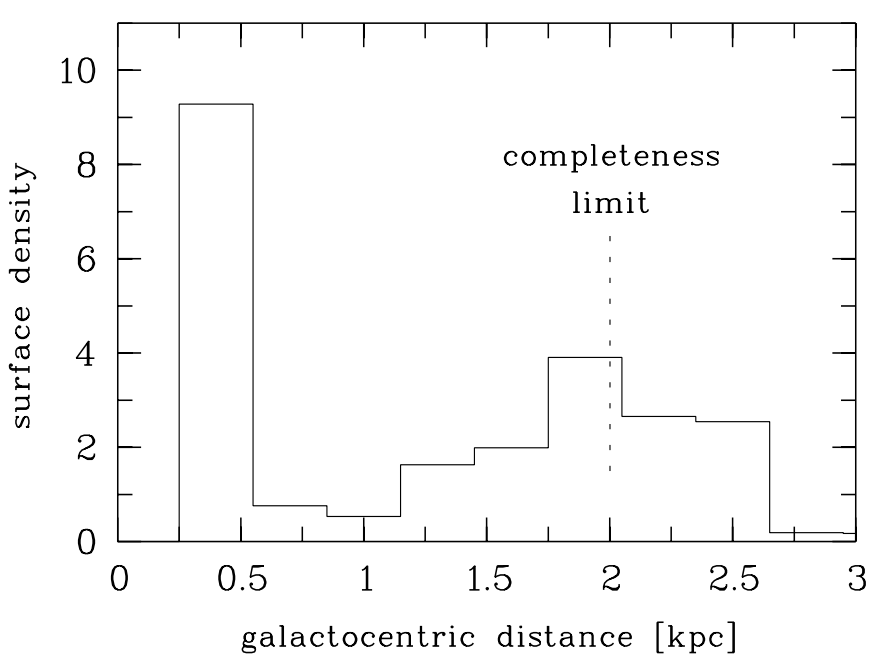

Fig. 7. Surface density of WR stars $/ \mathrm{kpc}^{2}$ in the inner region of NGC 300. The completeness limit is reached at $2 \mathrm{kpc}\left(\rho / \rho_{\mathrm{O}}=0.4\right)$, since our imaging was not centred on the nucleus.

The continuum filter centred at $4781 \AA$ lies midway between the usual Smith (1968) WR narrow-band $b(4270 \AA)$ and $v$ $(5160 \AA)$ filters. Typical intrinsic colours of WC and WNE stars are $(b-v)_{0} \sim-0.2 \pm 0.1$, such that one would expect $b-v \sim-0.1 \pm 0.1 \mathrm{mag}$ for WR stars in NGC 300, given typical extinctions of $E(b-v) \sim 0.1 \mathrm{mag}$ (equivalent to $E(B-V)=0.12 \mathrm{mag})$. Consequently, continuum filter measurements should correspond closely (within $\sim 0.1 \mathrm{mag}$ ) to $b$ or $v$ magnitudes. I.e. a completeness to $v=23.7$ mag will be equivalent to $M_{v} \sim-3.2 \mathrm{mag}$. According to Table 28 from van der Hucht (2001), $94 \%$ of the 227 known Galactic WR stars are brighter than $M_{v}=-3.5 \mathrm{mag}$, so our census of the central region of NGC 300 should be reasonably complete, except those suffering from high visual extinction.

In Fig. 7 we plot the WR surface density versus the galactocentric distance. The WR distribution in the nuclear region is particularly interesting. While the very centre is apparently free of WR stars we find a sharp increase of the surface density at a galactocentric distance of about $0.4 \mathrm{kpc}$. Further outside it drops first to a minimum at around $1 \mathrm{kpc}$ and rises again outwards. Qualitatively, a similar behaviour is observed in our Galaxy (van der Hucht 2001) but in NGC 300 the drop is much shallower, about -0.3 dex between 0.5 and $2 \mathrm{kpc}$ instead of -1.5 dex in the galaxy. The highest surface density in NGC 300 occurs in the Deh 137 HII region, alias OB association AS 102 which contains $15 \mathrm{WR}$ stars in an area that spans $0.3 \times 0.3 \mathrm{kpc}$ implying a WR density of about $150 \mathrm{WR}$ stars $/ \mathrm{kpc}^{2}$. Massey \& Johnson (1998) compare WR surface densities of other Local Group galaxies, such that WR surface densities range from $1 / \mathrm{kpc}^{2}$ in the SMC, to 2 in the LMC and $\sim 4$ in M 33.

As discussed in the introduction, the $\mathrm{WC} / \mathrm{WN}$ ratio for NGC 300 prior to the present study, i.e. 2, was unusually high relative to more complete surveys of Local Group galaxies. Ideally, one might use additional narrow band filters at $\left(\mathrm{C}_{\mathrm{IV}}\right)$ $\lambda \lambda 5801-12$ plus a nearby continuum region to discriminate between WN and WC stars, as recently carried out by Royer et al. (2001) for IC10. In the absence of such filters for FORS2, 


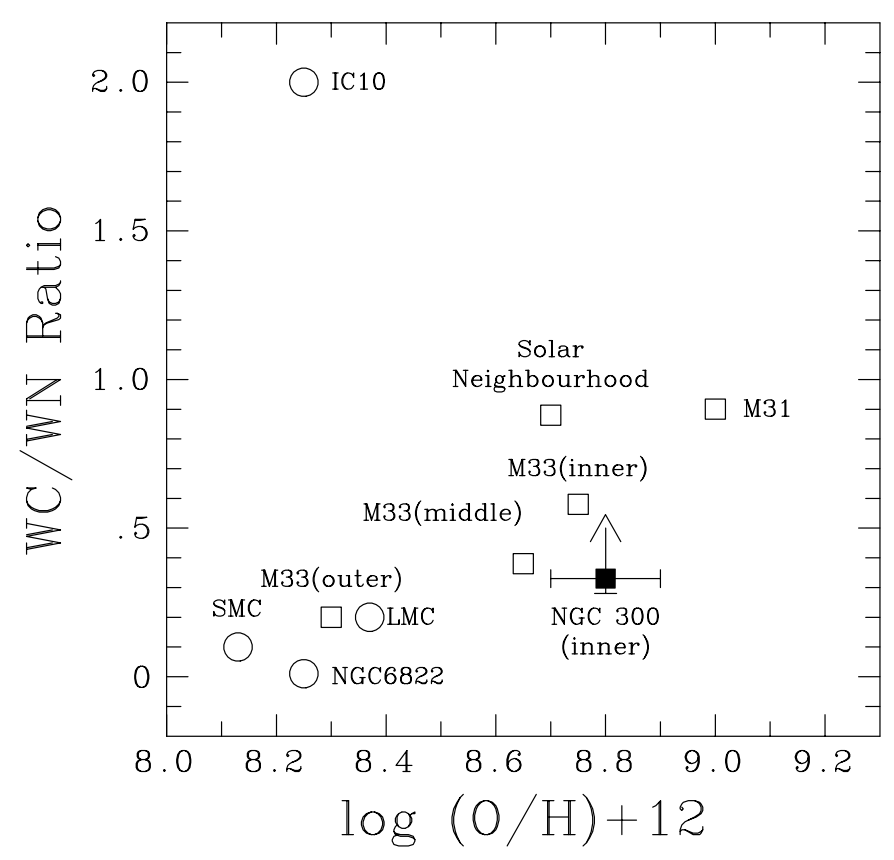

Fig. 8. The relative number of $\mathrm{WC}$ and $\mathrm{WN}$ stars in Local Group galaxies versus metallicity (Massey 2003), supplemented by NGC 300 (solid) from the present work. The WC/WN ratio for IC10 is probably an overestimate.

we have been able to infer likely WN or WC subtypes for those stars without spectroscopy from inspection of Fig. 6. We suggest that at least 13 WR stars in NGC 300 are WC stars, i.e. 12 for which spectroscopy is available, plus \#5. A further 3 may host WC binaries, namely \#12,\#31, \#33, such that the WC/WN ratio for the central regions of NGC 300 is $\geq 12 / 46=0.3$, or more likely $\sim 15 / 43=0.35$. This falls close to that observed in comparable regions of M 33, according to Massey (2003), as illustrated in Fig. 8.

\section{Spectroscopy of Wolf-Rayet stars}

\subsection{Previously identified WR stars}

We present flux calibrated low dispersion FORS2 spectroscopy of four previously observed NGC $300 \mathrm{WC}$ stars in Fig. 9. These datasets are superior to previous $4 \mathrm{~m}$ observations, and so allow us to obtain revised spectral types, using the scheme of Crowther et al. (1998).

We revise the original classification for \#29, alias WR3 (Schild \& Testor 1991), from WC4-5 to WC5 since our dataset reveals weak C III $\lambda 5696$, with $W_{\lambda}(\lambda 5696) / W_{\lambda}(\lambda \lambda 5801-12) \sim$ 0.1 and $\mathrm{O}_{\text {III }} \mathrm{v} \lambda 5592$ weak/absent. This star is probably single, given that its emission line spectrum is comparable in strength (e.g. $\left.W_{\lambda}(\lambda \lambda 5801-12) \sim 800 \AA\right)$ to apparently single Galactic and LMC WCE stars.

A firm classification is possible for \#40, alias WR6 (Schild $\&$ Testor 1992) for which we also assign WC5 (updated from WC4-6) since C III $\lambda 5696$ is again present, with a similar strength to $\mathrm{O}$ III-V $\lambda 5592$ and $W_{\lambda}(\lambda 5696) / W_{\lambda}(\lambda \lambda 5801-12) \sim$ 0.1 . \#40 is almost certainly multiple, since $W_{\lambda}(\lambda 5801-12) \sim$ $230 \AA$.
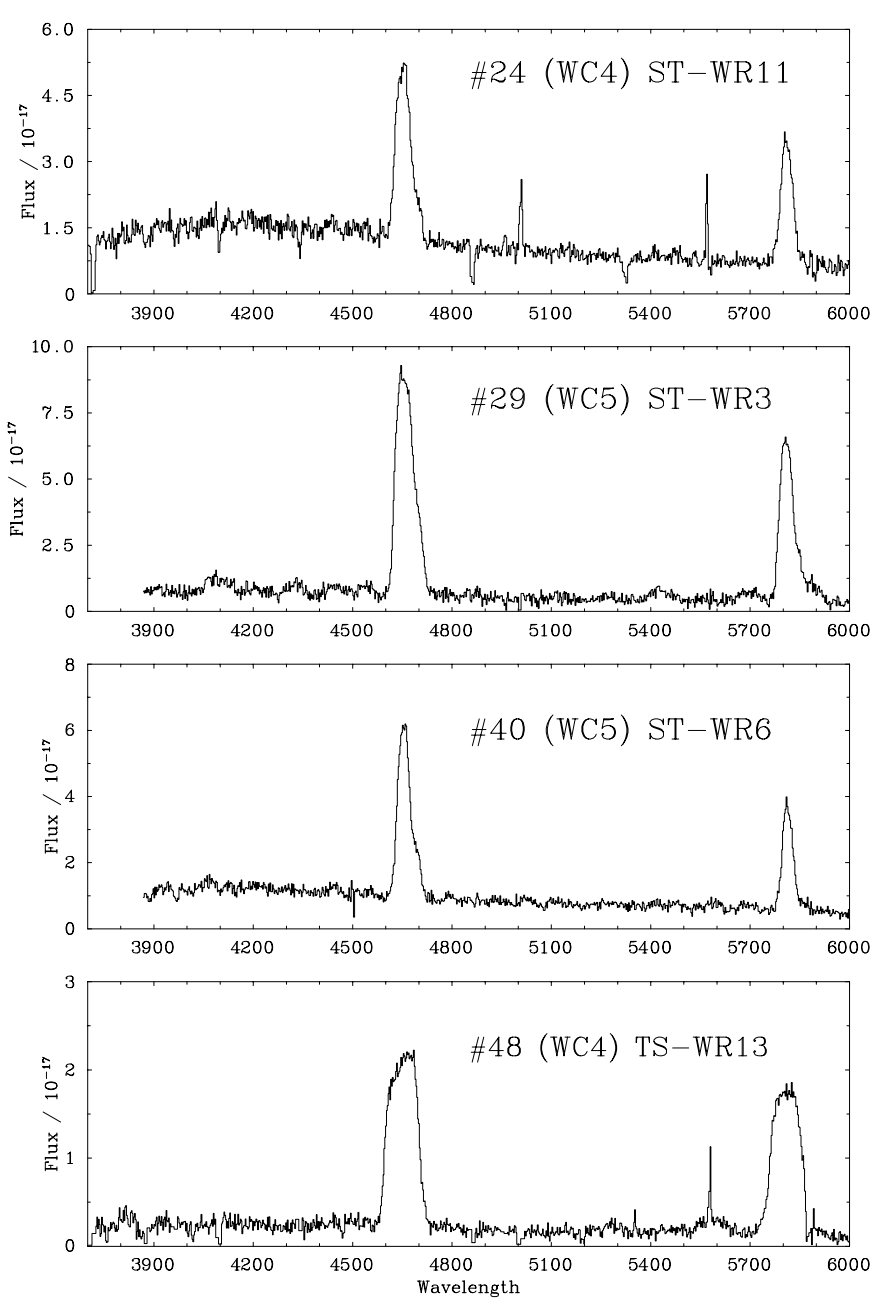

Fig. 9. Low dispersion FORS2 spectroscopy of four previously identified WR stars in NGC 300.

Testor \& Schild (1993) previously assigned a WC5 spectral type for \#24 (their WR11), which we revise to WC4, given that $W_{\lambda}(\lambda 5696) / W_{\lambda}(\lambda \lambda 5801-12) \leq 0.05 . \mathrm{C}$ IV $\lambda 5801-12$ is again unusually weak, with $W_{\lambda}(\lambda 5801-12) \sim 200 \AA$ indicating either binarity or a line-of-sight companion.

The spectral appearance of \#48, alias WR13 (Testor \& Schild 1993), alias IV-3 (Breysacher et al. 1997) is in marked contrast to the other WCE stars whose spectroscopy is presented here, with much broader lines - FWHM( $\lambda \lambda 5801-12) \sim$ $86 \AA$ versus $36-47 \AA$. Breysacher et al. (1997) interpreted this large $F W H M$ as an indication of a (rare) WO subtype, which possess strong $\mathrm{O}$ vi $\lambda \lambda 3811-34$ emission lines, and assigned a WO4 spectral type, whilst our spectroscopy reveals that O VI is weak/absent in \#48. Since C III $\lambda 5696$ is also absent, a WC4 spectral type is appropriate. Willis et al. (1992) discuss problems with using $F W H M$ as indicators of spectral type for WC stars in M 33. We suspect that \#48 is single since $W_{\lambda}(\lambda \lambda 5801-12) \sim 1500 \AA$.

\subsection{Newly identified WR stars}

We present optical spectroscopy of four newly identified NGC 300 WR stars in Fig. 10, two WN and two WC stars. 

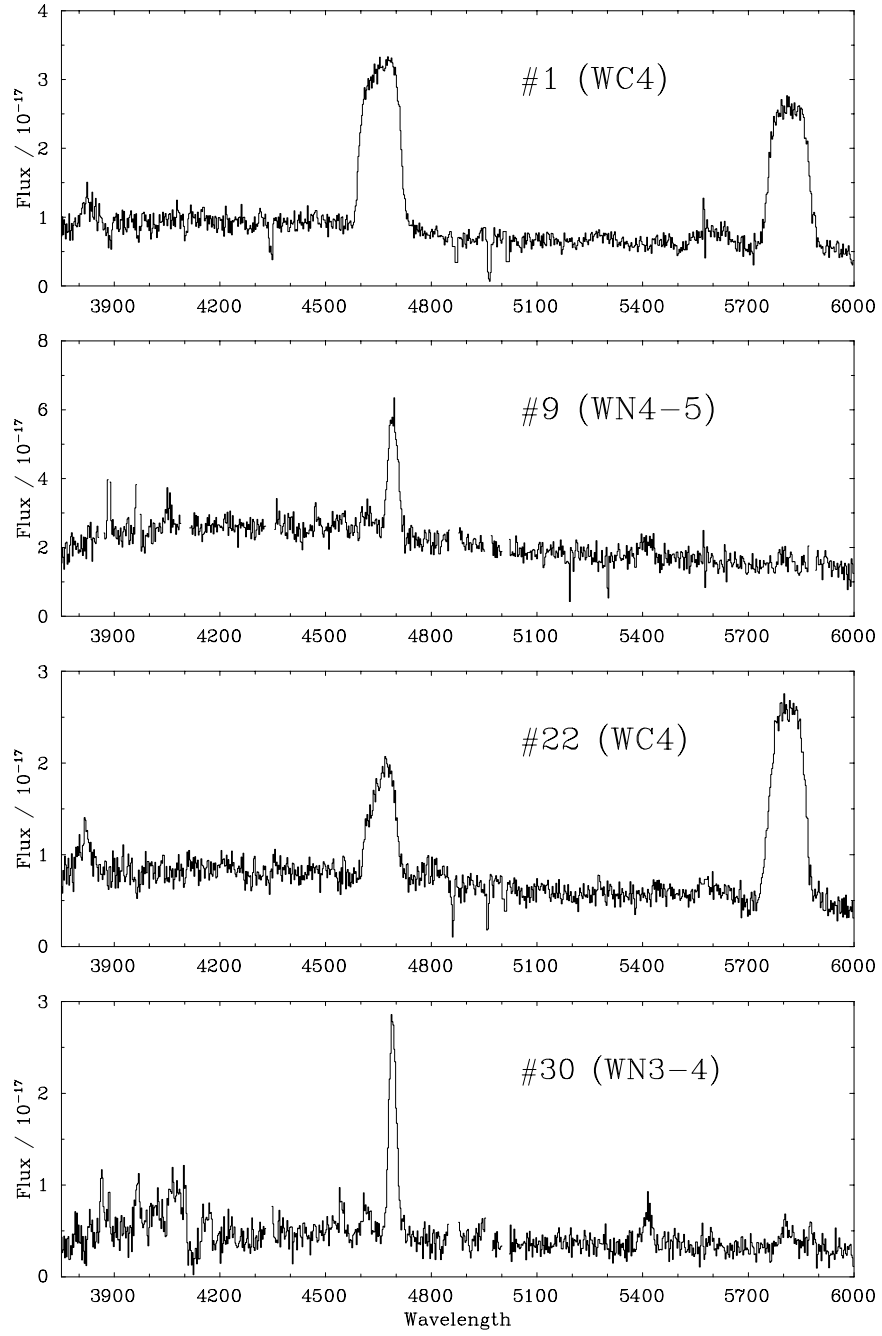

Fig. 10. Low dispersion FORS2 spectroscopy of four newly identified WR stars in NGC 300.

The WC stars \#1 and \#22 are rather similar. They have lines widths which are higher than \#48, with $F W H M(\lambda \lambda 5801-12) \sim$ 91-100 Å, although lower line strengths, $W_{\lambda}(\lambda \lambda 5801-12) \sim$ $500 \AA$. WC4 subtypes are appropriate for both stars since there is no evidence of $\mathrm{C}_{\mathrm{III}} \lambda 5696$, with $\mathrm{O}$ vI $\lambda \lambda 3811-34$ weak. Both stars are probably binaries.

The two WN stars \#9 and \#30 are early-type, since $\mathrm{Nv} \lambda \lambda 4603-20$ is prominent, with $\mathrm{N}_{\text {III }} \lambda \lambda 4634-41$ weak (\#9) or absent (\#30). Following the classification scheme of Smith et al. (1998) one obtains a spectral type of WN4-5 for \#9 (N Iv $\lambda 4058 \sim \mathrm{N} v \lambda \lambda 4603-20$ ), and WN3-4 for \#30 (the region around $\mathrm{N}$ IV $\lambda 4058$ is noisy). One cannot use the (primary) He I-II classification diagnostics for these stars due to the strong nebular contamination, and weak He I $\lambda 5876$ emission.

\subsection{WC line width}

Willis et al. (1992) identified a correlation between line width (FWHM C IV $\lambda \lambda 5801-12$ ) and galactocentric distance for WCE stars in M 33 in the sense that stars at larger galactocentric distance (i.e. lower metallicity) had broader lines than those in the nucleus (with higher metallicity). We present our

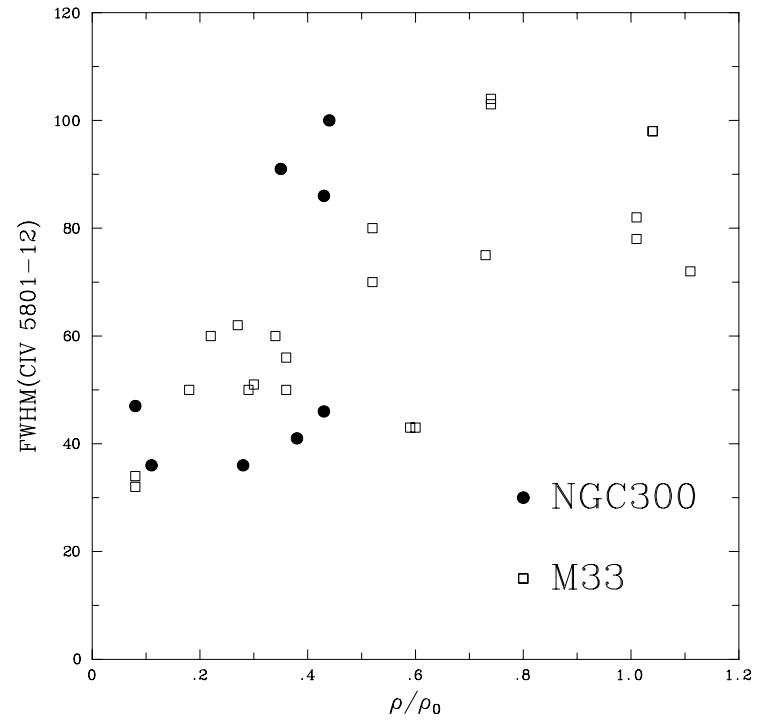

Fig. 11. Comparison between $F W H M\left(\mathrm{C}_{\text {IV }} \lambda \lambda 5801-12\right)$, in $\AA$, and galactocentric distance, as a fraction of the Holmberg radius, for early WC stars in NGC 300 (solid) and M 33 (open, Willis et al. 1992).

measurements for $6 \mathrm{WC}$ stars in NGC 300 in Fig. 11, supplemented by data from Schild \& Testor $(1991,1992)$ for \#14 (WR1) and \#47 (WR5), and including data from Willis et al. (1992) and references therein for M 33. For NGC 300, there is a very large scatter in $F W H M$ at $\rho / \rho_{0} \sim 0.4$, arguing against a tight correlation in general, although the present results are in favour of a deficit of broad-lined WC stars in the nucleus. Nevertheless, firm conclusions await spectroscopy of larger numbers of WC stars in both galaxies.

\section{Analysis of WC stars}

As discussed above in Sect. 4, two WC stars in NGC 300 are apparently single, and have sufficient quality observations for detailed analyses to be carried out. Ultimately, large numbers of WR stars need to be studied in galaxies spanning a wide range metallicities to place adequate constraints on evolutionary models. Recent studies, using identical techniques, have been presented for single WC stars in the Milky Way (e.g. Dessart et al. 2000), LMC (Crowther et al. 2002), M 31 (Smartt et al. 2001) and M 33 (Abbott et al. 2003). We now proceed to study \#29 (WC5), located close to the nucleus of NGC 300 with a probable metallicity of $\sim Z_{\odot}$ according to $10^{4} \mathrm{O} / \mathrm{H}=$ 7.5-5.3 $\rho / \rho_{0}$ (Deharveng et al. 1988), and \#48 (WC4), located at $\rho / \rho_{0}=0.43$ with $\sim 0.6 Z_{\odot}$.

\subsection{Technique}

We employ the non-LTE code of Hillier \& Miller (1998), which iteratively solves the transfer equation in the co-moving frame subject to statistical and radiative equilibria in an expanding, spherically symmetric and steady-state atmosphere. Specific details of the (extremely complex) He, C, O, Ne, Si, P, S, Ar, $\mathrm{Fe}$ model atoms used for our quantitative analysis are provided 
in Crowther et al. (2002). We assume that the wind is clumped with a volume filling factor, $f \sim 0.1$. We parameterise the filling factor so that it approaches unity at small velocities.

As usual, a series of models were calculated in which stellar parameters $\left(T_{*}, \log L / L_{\odot}, v_{\infty} \dot{M} / \sqrt{f}, \mathrm{C} / \mathrm{He}, \mathrm{O} / \mathrm{He}\right)$ were adjusted until the observed ionization balance, line strengths, widths, and absolute $v$-band flux were reproduced. Because of the substantial effect that differing mass-loss rates, temperatures and elemental abundances have on the emergent spectrum, this was an iterative process. Abundances other than He, $\mathrm{C}$ and $\mathrm{O}$, namely $\mathrm{Ne} \ldots \mathrm{Fe}$, were set at $0.5 Z_{\odot}$, although the exact choice does not greatly affect the emergent spectrum (Crowther et al. 2002). As discussed above, we select $v \sim m_{4781}$ for both stars. Despite the narrow spectral range available, we derive a reddening of $E_{b-v}=0.08 \mathrm{mag}\left(E_{B-V}=0.10 \mathrm{mag}\right)$ for \#29 and $E_{b-v}=0.12 \mathrm{mag}\left(E_{B-V}=0.15 \mathrm{mag}\right)$ for \#48, in good agreement with $\mathrm{H}_{\text {II }}$ regions (Deharveng et al. 1988). A standard Galactic extinction law of Seaton (1979) is adopted. Accounting for uncertainties in $v$, distance and reddening, we obtain $M_{v}=-4.6 \pm 0.2 \mathrm{mag}$ for $\# 29$ and $M_{v}=-3.5 \pm 0.2 \mathrm{mag}$ for \#48.

The wind ionization balance is ideally selected on the basis of isolated optical lines from adjacent ionization stages of carbon and/or helium, e.g. He I $\lambda 5876 / \mathrm{He}$ II $\lambda 5412$. In practice, this was difficult to achieve because of the severe blending, so our derived temperature should be treated as approximate. Detection of He I $\lambda 5876$ appears to be robust in \#29, due to its relatively low wind velocity, whilst there is an ambiguity in this feature for \#48, since it is possible that the observed feature represents the electron scattering wing of $\mathrm{C}_{\mathrm{IV}}$ for which we have adopted the filling factor, $f$. We also simultaneously match $C_{\text {III }} \lambda 6740$ and $C_{\text {IV }} \lambda 5801$, the former selected in preference to $C_{\text {III }} \lambda 5696$ which is very sensitive to the exact ionization structure (Hillier \& Miller 1998; Crowther et al. 2002). The standard C/He diagnostic, He II $\lambda 5412 / \mathrm{C}_{\text {IV }} \lambda$ 15471, was used since their relative strengths are insensitive to temperature and mass-loss. Oxygen abundances were difficult to constrain, since we relied solely on O III-V $\lambda 5592$ (Crowther et al. 2002). Consequently, caution is advised when comparing the present $\mathrm{O} / \mathrm{C}$ determinations with (Galactic and LMC) WC stars for which the superior $\lambda \lambda 2800-3100$ diagnostics are available.

\subsection{Results for NGC300 \#29 (WR3, WC5)}

Our FORS2 spectroscopic data of \#29 is shown in the upper panel of Fig. 12. Overall, the spectrum is reasonably well reproduced by our model fit, except that the C III $\lambda 5696$ profile is strongly underestimated, whilst C IV $\lambda \lambda 5801-12$ is $40 \%$ too weak. From our recent experience it is difficult to simultaneously reproduce the strength of $\mathrm{C}_{\text {III }} \lambda 5696$ feature together with other diagnostics in early WC stars. We find $T_{*} \sim 100 \mathrm{kK}$, $\log \left(L / L_{\odot}\right)=5.5, v_{\infty} \sim 2700 \mathrm{~km} \mathrm{~s}^{-1}$, and $\dot{M} \sim 10^{-4.6} M_{\odot} \mathrm{yr}^{-1}$. We estimate $\mathrm{C} / \mathrm{He} \sim 0.08$ by number from He II $\lambda 5412 / \mathrm{C}$ IV 15471. The weak O III-V $\lambda 5592$ feature suggests a low oxygen content of $\mathrm{O} / \mathrm{He} \leq 0.05$ by number.
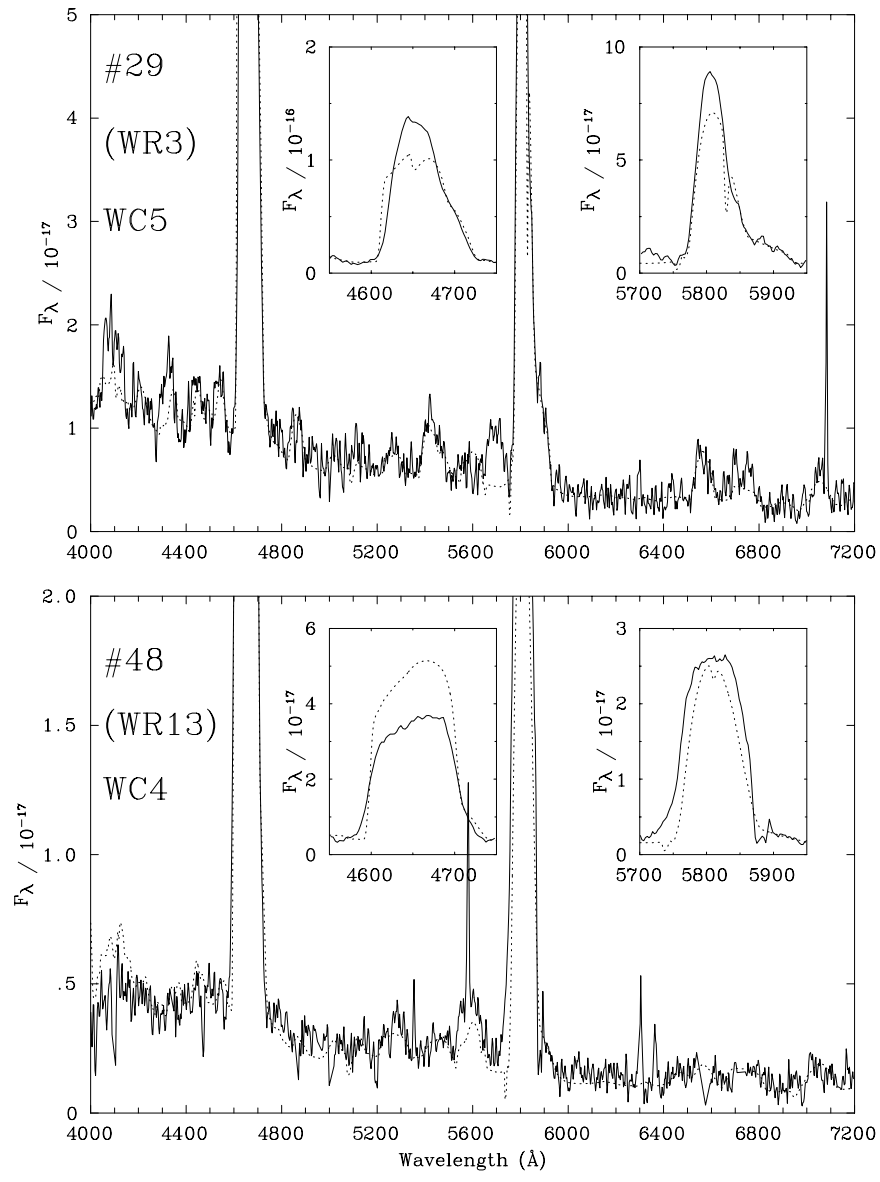

Fig. 12. Upper panel: Synthetic spectral fit (dotted) to FORS2 observations (solid) of NGC 300 \#29 (WR3, WC5), de-reddened by $E_{B-V}=0.10$ mag. Close up views of the C III-IV-He II $\lambda \lambda 4650-4686$ and $C_{\text {IV }} \lambda \lambda 5801-12$ regions are indicated. Lower panel: Same for NGC $300 \# 48$ (WR13, WC4) for a reddening of $E_{B-V}=0.15 \mathrm{mag}$.

\subsection{Results for NGC $300 \# 48$ (WR13, WC4)}

We compare our spectroscopy of \#48 with our synthetic model in the lower panel of Fig. 12. Again, reasonably good agreement is achieved, although the broad emission lines of \#48 hinder detailed comparisons. The blend comprising principally $\mathrm{C}_{\text {III }} \lambda 4647-51, \mathrm{C}_{\text {IV }} \lambda 4660$ and He II $\lambda 4686$ is rather too strong in the synthetic model. Our derived parameters are $T_{*} \sim 95 \mathrm{kK}$, $\log \left(L / L_{\odot}\right)=5.2, v_{\infty} \sim 3750 \mathrm{~km} \mathrm{~s}^{-1}$, and $\dot{M} \sim 10^{-4.8} M_{\odot} \mathrm{yr}^{-1}$. We estimate $\mathrm{C} / \mathrm{He} \sim 0.5$ by number, although this ratio should be treated with caution, given the poor quality of the observations - recall \#48 has the faintest continuum $(v=23.5 \mathrm{mag})$ of all 58 WR candidates in NGC 300. The O III-V $\lambda 5592$ feature suggests a high oxygen content of $\mathrm{O} / \mathrm{He} \geq 0.1$ by number.

\subsection{Comparison with WC stars in the Galaxy and $L M C$}

Crowther et al. (2002) recently contrasted the properties of Solar neighbourhood and LMC WC stars, to which we can now add NGC 300 \#29 and \#48. The upper panel in Fig. 13 compares (nuclear) luminosities and $(\mathrm{C}+\mathrm{O}) / \mathrm{He}$ abundances for WC stars in the three galaxies. Nuclear luminosities are 

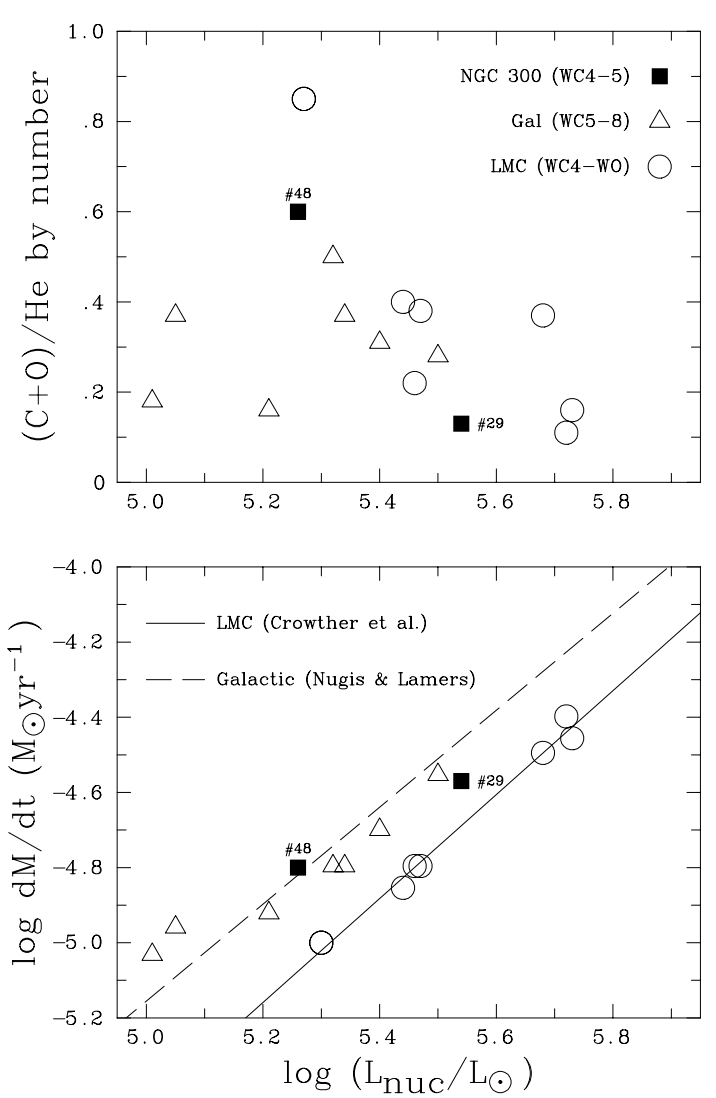

Fig. 13. Upper panel: comparison between the $(\mathrm{C}+\mathrm{O}) / \mathrm{He}$ ratios of WC stars in NGC 300 (filled symbols, this work), the Milky Way and LMC (see Crowther et al. 2002), as indicated in the key; Lower panel: comparison between the mass-loss rates and luminosities of NGC 300 (filled symbols, this work), Milky Way and LMC WC stars (see Crowther et al. 2002), as indicated above. A fit to the LMC WC4 stars (solid) is indicated, as is the generic Galactic WR mass-loss calibration from Nugis \& Lamers (2000), assuming $\mathrm{C} / \mathrm{He}=0.2, \mathrm{C} / \mathrm{O}=4$ by number.

derived by taking into account the wind blanketing effects discussed by Heger \& Langer (1996). In contrast with the results of Heger \& Langer, who indicated revisions of up to $0.3 \mathrm{dex}$ in luminosity, revised mass-loss rates due to clumping yield rather small corrections, typically 0.05 dex. Current masses of 16.3 and $11.6 M_{\odot}$ are determined for \#29 and \#48, respectively. Crowther et al. (2002) found that low metallicity (LMC) WC stars possess higher luminosities than those at high metallicity (Milky Way). This can be explained since one would require a higher initial mass cut-off, for a massive star to progress through to the WC stage at low metallicity, because of reduced mass-loss rates during the main-sequence and post-main sequence evolution. The small sample of NGC 300 stars does not allow firm conclusions to be drawn, given that they span a range in luminosity common to LMC and Galactic WC stars.

Figure 13 suggests that \#48 has a high (albeit uncertain) metal content, whilst \#29 has a $(\mathrm{C}+\mathrm{O}) / \mathrm{He}$ ratio which is amongst the lowest of WC stars studied to date. Analyses of larger numbers of WC stars in external galaxies is urgently required for general trends to be identified.
Crowther et al. (2002) also identified a trend towards weaker winds for WC stars at lower metallicities, such that $\dot{M} \propto Z^{0.5}$, which explained the relative WC subtype distributions in the LMC and Galaxy. The lower panel of Fig. 13 compares luminosities and mass-loss rates of Galactic, LMC and NGC $300 \mathrm{WC}$ stars, including a fit to the LMC data (from Crowther et al.) plus the Galactic calibration of Nugis \& Lamers (2000). Both \#29 (WC5) and \#48 (WC4) lie on, or slightly below, the Galactic calibration, as would be expected from the metallicities inferred from their locations in NGC 300 (Deharveng et al. 1988; Zaritsky et al. 1994). Of course, the difference between Galactic and LMC calibrations amounts to less than a factor of two in mass-loss rate, and Smartt et al. (2001) discuss, in detail, limitations with adopting generic $\mathrm{H}_{\text {II }}$ region abundances with regard to WC properties in M 31 .

\section{Conclusions}

We have demonstrated the feasibility of using narrow-band filters to detect Wolf-Rayet candidates in NGC 300. Restricting our survey to the central $6.8^{\prime} \times 6.8^{\prime}$ region, we have trebled the known WR content from 20 to 58 stars, within a factor of two of the global content of its northern Local Group counterpart, M 33. Surveys of the outer spiral arms of NGC 300 are sought in order to determine its total WR content, which probably approaches $\sim 100$, as is spectroscopic confirmation of remaining candidates. The $\mathrm{WC} / \mathrm{WN}$ ratio of the central region of NGC 300 has been revised from $\sim 2$ to $\sim 1 / 3$, in reasonable accord with Local Group galaxies spanning a similar metallicity range. Modern abundance analyses of $\mathrm{H}$ II regions and/or $\mathrm{AB}$ supergiants are urgently required to verify previous determinations of the metallicity gradient of NGC 300 . We have purposefully not discussed the WR/O ratio in NGC 300, since it is extremely difficult to constrain this ratio observationally, as discussed by Massey (2003).

Using VLT-FORS2, $600 \mathrm{~s}$ imaging provides over $90 \%$ completeness for WR candidates down to an excess of $0.1 \mathrm{mag}$ at a distance of $2 \mathrm{Mpc}$. The number of known WR stars can therefore be rapidly increased with a modest investment of observing time. This approach greatly improves our chance of witnessing a WR star undergoing a supernova explosion in the nearby universe. Imaging surveys towards such goals are presently underway (e.g. Smartt et al. 2002), albeit based solely around broad-band filters, such that WR candidates can not easily be identified. Under favourable conditions one can reasonably expect to extend our imaging/spectroscopic approach to WR stars within galaxies at distances of up to at least $\sim 5 \mathrm{Mpc}$. Recession radial velocities, shifting WR emission lines redward of the $\lambda 4684$ filter, only become problematic in excess of $1000 \mathrm{~km} \mathrm{~s}^{-1}$, typically corresponding to distances in excess of $\geq 10 \mathrm{Mpc}$. We have recently obtained VLT-FORS2 narrow-band imaging of the metal-rich spiral galaxy M 83 (NGC 5236), at distance of $3.2 \mathrm{Mpc}^{2}$ (Freedman et al. 2001). M 83 has a metallicity of up to $12+\log (\mathrm{O} / \mathrm{H})=9.2$ (Bresolin \& Kennicutt 2002), such that a large WR population is expected.

\footnotetext{
${ }^{2}$ We assume that its distance is comparable to NGC 5253.
} 
Once the census of WR stars is reasonably complete in a galaxy or part thereof, we can obtain surface density plots. We present here the WR star distribution in the central $2 \mathrm{kpc}$ of NGC 300. We find that the very centre of the galaxy is apparently void of WR stars, in contrast with our own Galaxy, but that a maximum of the surface density occurs at a galactocentric distance of about $0.4 \mathrm{kpc}$. At $1 \mathrm{kpc}$ the surface density drops to a minimum, beyond which it steadily increases to about half the value of the $0.5 \mathrm{kpc}$ ring. NGC 300 compares favourably with most Local Group galaxies in its WR surface density, with the exception of IC10, and perhaps M 33 (Massey \& Johnson 1998).

We have also illustrated that single, early-type WC stars with $v \sim 23$ mag can be quantitatively studied using modest integration times with VLT-FORS2. More problematic is the challenge of obtaining uncontaminated WR spectroscopy at such large distances, since a slit width of $1^{\prime \prime}$ corresponds to a spatial scale of $\sim 10 \mathrm{pc}$ at $2 \mathrm{Mpc}$. Isolated WR stars are present, although they are in the minority and will be even more problematic for still more distant galaxies. An order-of-magnitude reduction in slit size is ultimately required using ground-based telescopes, without a corresponding loss of throughput.

Acknowledgements. Thanks to John Hillier for the generous use of his model atmosphere code, to the Conception group for providing photometric standards in advance of publication, and to Mischa Schirmer for forwarding the WFI image of NGC 300. PAC acknowledges financial support from the Royal Society. The routine to determine deprojected galactocentric distances was adapted from fortran code provided by Phil Massey.

\section{References}

Abbott, J. B., Crowther, P. A., Drissen, L., et al. 2003, in Proc. IAU Symp. 212, ed. K. A. van der Hucht et al., ASP, in press

Bohannan, B., \& Crowther, P. A. 1999, ApJ, 511, 374

Bresolin, F., \& Kennicutt, R. C. Jr 2002, ApJ, 572, 838

Bresolin, F., Gieren, W., Kudritzki, R. P., Pietrzyński, G., \& Przybilla, N. 2002a, ApJ, 567, 277

Bresolin, F., Kudritzki, R. P., Najarro, F., Gieren, W., \& Pietrzyński, G. 2002b, ApJ, 577, L107
Breysacher, J., Azzopardi, M., Testor, G., \& Muratorio, G. 1997, A\&A, 326, 976

Crowther, P. A. 2000, A\&A, 356, 191

Crowther, P. A., De Marco, O., \& Barlow, M. J. 1998, MNRAS, 296, 367

Crowther, P. A., Dessart, L., Hillier, D. J., et al. 2002, A\&A, 392, 653

Deharveng, L., Caplan, J., Lequeux, J., et al. 1988, A\&AS, 73, 407

Dessart, L., Crowther, P. A., Hillier, D. J., et al. 2000, MNRAS, 315, 407

D’Odorico, S., Rosa, M., \& Wampler, E. J. 1983, A\&AS, 53, 97

Freedman, W. L., Madore, B. F., Gibson, B. K., et al. 2001, ApJ, 553, 47

Heger, A., \& Langer, N. 1996, A\&A, 315, 421

Hillier, D. J., \& Miller, D. L. 1998, ApJ, 496, 407

Massey, P. 1996, in Proc. 33rd Liège Intl. Astrophysics Coll., 361

Massey, P. 2003, in Proc. IAU Symp. 212, ed. K. A. van der Hucht et al., ASP, in press

Massey, P., \& Johnson, O. 1998, ApJ, 505, 793

Nugis, T., \& Lamers, H. J. G. L. M. 2000, A\&A, 360, 227

Pagel, B. E. J., Edmunds, M. G., Blackwell, D. E., Chun, M. S., \& Smith, G. 1979, MNRAS, 189, 95

Pannuti, T. G., Durie, N., Lacey, C. K., et al. 2001, ApJ, 544, 780

Pietrzyński, G., Gieren, W., Fouqé, P., \& Pont, F. 2001, A\&A, 371, 497

Pietrzyński, G., Gieren, W., \& Udalski, A. 2002a, PASP, 114, 298

Pietrzyński, G., Gieren, W., Fouqé, P., \& Pont, F. 2002b, AJ, 123, 789

Royer, P., Smartt, S. J., Manfroid, J., \& Vreux, J.-M. 2001, A\&A, 366, L1

Schild, H., \& Testor, G. 1991, A\&A, 243, 115

Schild, H., \& Testor, G. 1992, A\&A, 266, 145

Seaton, M. J. 1979, MNRAS, 187, 73

Smartt, S. J., Crowther, P. A., Dufton, P. L., et al. 2001, MNRAS, 325, 257

Smartt, S. J., Vreeswijk, P. M., Ramirez-Ruiz, E., et al. 2002, ApJ, 572, L147

Smith, L. F. 1968, MNRAS, 140, 409

Smith, L. F., Shara, M. M., \& Moffat, A. F. J. 1996, MNRAS, 281, 163

Testor, G., \& Schild, H. 1993, ESO Messenger, 72, 31

van der Hucht, K. A. 2001, New Astron. Rev., 45, 135

Webster, B. L., \& Smith, M. G. 1983, MNRAS, 204, 743

Willis, A. J., Schild, H., \& Smith, L. J. 1992, A\&A, 261, 419

Zaritsky, D., Kennicutt, R. C. Jr, \& Huchra, J. P. 1994, ApJ, 420, 87 